\title{
Exchange Stiffness of Ferromagnets
}

\author{
M.D. Kuz'min, ${ }^{1}$ K.P. Skokov ${ }^{2}$ L.V.B. Diop, ${ }^{2}$ I.A. Radulov, ${ }^{2}$ and O. Gutfleisch ${ }^{2}$ \\ ${ }^{1}$ Aix-Marseille Université, IM2NP, UMR CNRS 7334, 13397 Marseille, France and \\ ${ }^{2}$ Institut für Materialwissenschaft, Technische Universität Darmstadt, D-64287 Darmstadt, Germany
}

(Dated: April 12, 2018)

\begin{abstract}
Exchange stiffness constants of 22 ferromagnetic compounds have been deduced from their spontaneous magnetization, $M_{s}$, by using an improved technique. The improvement consists in utilizing the entire $M_{s}(T)$ curve, up to the Curie point, rather than just its low-temperature part, with $T \ll T_{C}$. For 17 of the 22 ferromagnets literature data have been used, while 5 compounds have been studied anew, on single crystals grown for the purpose.
\end{abstract}

Exchange stiffness is the prefactor of the gradient term in the energy density of an inhomogeneously magnetized ferromagnet,

$$
E=A\left(\left|\nabla m_{x}\right|^{2}+\left|\nabla m_{y}\right|^{2}+\left|\nabla m_{z}\right|^{2}\right),
$$

where $\boldsymbol{m}$ is the unit vector in the direction of magnetization. $A$ is a necessary ingredient of any micromagnetic calculation, the applications ranging from the coercivity of bulk materials to nanoscopic devices such as the racetrack memory. ${ }^{1}$

A way to determine $A$ was proposed by Landau and put in writing by Lifshitz. ${ }^{2}$ In this method one first finds the spin-wave stiffness $D$ (i.e., the prefactor in the magnon dispersion relation, $\hbar \omega_{q}=D q^{2}$ ) by fitting a measured temperature dependence of spontaneous magnetization to Bloch's law, ${ }^{3}$

$$
M_{s}(T)=M_{0}\left[1-0.0586 \frac{g \mu_{B}}{M_{0}}\left(\frac{k T}{D}\right)^{3 / 2}\right] .
$$

Here $M_{0}$ is (volume) saturation magnetization. Knowing $D$, one then finds $A$ from a simple proportionality relation that exists between both quantities: ${ }^{2}$

$$
A=\frac{M_{0}}{2 g \mu_{B}} D
$$

The advantageous, model-independent character of this relation was pointed out by Herring and Kittel. ${ }^{4}$ Later, in the light of the density functional theory (DFT), it was realized that the decisive advantage of Eq. (3) is that $A, D$, and the proportionality factor - equal to one-half of the mean spin density - are all ground-state properties. (Our consideration is limited to materials where orbital contribution to $M_{0}$ can be neglected and, therefore, $g=2$.)

One difficulty of the Landau-Lifshitz technique is that the Bloch law (2) is only valid at low temperature (strictly speaking, infinitesimally low) and it is from the infinitesimally small difference between $M_{s}$ and $M_{0}$ that $D$ has to be extracted.

Other methods have their own difficulties and as a result our knowledge of $A$ and $D$ remains inaccurate. Thus, the determination of $A$ from the Curie temperature, $T_{C}$, is based essentially on the localized (Heisenberg) model with nearest-neighbor exchange. Another technique, inelastic neutron scattering on magnons, purports to provide a direct access to $D$. Yet, the simple quadratic form, $\hbar \omega_{q}=D q^{2}$, is not valid but for $q$ small, and that part of the spectrum is never observed. Extrapolation from higher wavenumbers/energies leads to ambiguity, cf. the results for Ni in Table II. A detailed review of the various techniques and values of $A$ was given by Döring over halfa-century ago (Ref. 5, Sections 21-28). The situation has ameliorated little since then.

In this Letter we present an improved method of determining $A$, where the first stage consists in fitting the entire $M_{s}(T)$ curve, with $0<T<T_{C}$, to an approximate expression, ${ }^{6}$

$$
M_{s}(T)=M_{0}\left[1-s\left(\frac{T}{T_{C}}\right)^{3 / 2}-(1-s)\left(\frac{T}{T_{C}}\right)^{p}\right]^{\beta},
$$

where $p=5 / 2, \beta=1 / 3$, and $s$ is an adjustable parameter. In the low-temperature limit Eq. (4) turns into the Bloch law, $M_{s} \approx M_{0}\left[1-s \beta\left(T / T_{C}\right)^{3 / 2}\right]$, whence

$$
D=0.1509\left(\frac{g \mu_{B}}{s \beta M_{0}}\right)^{2 / 3} k T_{C} \text {. }
$$

Thus, $A$ is found from the best-fit value of $s$ by using successively Eqs. (5) and (3). The advantage is that now all $M_{s}(T)$ data are used, not just those at $T \ll T_{C}$. The choice of the upper limit of the temperature interval presents no longer a problem and is no more a source of ambiguity.

For a demonstration of the new method we collected data on 17 ferromagnets available in the literature. These are presented in the upper part of Table I. For each compound a reference is given to the paper where the fitting to Eq. (4) was performed and where further references may be found. Two ferromagnets were reported to have non-standard values of the parameter $p$ : Fe $(p=4)$ and $\mathrm{MnB}(p=10)$; this fact does not affect in any way the determination of $D$ or $A$. As against that, for the three compounds where non-standard values of the critical exponent $\beta$ had been employed in the fit, ${ }^{14} \beta=0.369$ for EuS and $\mathrm{EuO}$ and $\beta=0.31$ for $\mathrm{CrBr}_{3}$, the same values had to be set in Eq. (5) in order to compute $D$. In all other cases standard parameter values were used, $p=5 / 2$ and $\beta=1 / 3$. 
TABLE I. Quantities relevant to the determination of exchange stiffness $A$.

\begin{tabular}{lcccccc}
\hline compound & $M_{0}(\mathrm{MA} / \mathrm{m})$ & $T_{C}(\mathrm{~K})$ & $s$ & $D\left(\mathrm{meV}^{2}\right)$ & $A(\mathrm{pJ} / \mathrm{m})$ & reference \\
\hline $\mathrm{MnB}$ & 0.99 & 567 & 0.7 & 140 & 5.9 & 7 \\
$\mathrm{Fe}_{2} \mathrm{~B}$ & 1.29 & 1005 & 0.9 & 170 & 9.6 & 8 \\
$\mathrm{Co}_{2} \mathrm{~B}$ & 0.55 & 417 & 0.5 & 190 & 4.4 & 8 \\
$\mathrm{La}_{2} \mathrm{Co}_{7}$ & 0.56 & 479 & 0.75 & 160 & 3.9 & 9 \\
$\mathrm{Y}_{2} \mathrm{Fe}_{14} \mathrm{~B}$ & 1.20 & 568 & 0.7 & 120 & 6.3 & 10 \\
$\mathrm{Y}_{2} \mathrm{Fe}_{14} \mathrm{Si}_{3}$ & 0.95 & 483 & 0.8 & 110 & 4.5 & 11 \\
$\mathrm{Lu}_{2} \mathrm{Co}_{17}$ & 1.06 & 1210 & 0.35 & 440 & 20 & 12 \\
$\mathrm{Gd}_{\mathrm{EuS}}$ & 2.12 & 289 & 1.3 & 28 & 2.5 & 13 \\
$\mathrm{EuO}$ & 1.22 & 16.6 & 0.8 & 3.0 & 0.16 & 14 \\
$\mathrm{CrBr}$ & 1.91 & 69.6 & 0.8 & 9.3 & 0.77 & 14 \\
$\mathrm{Fe}_{\mathrm{Co}}$ & 0.27 & 32.6 & 1.0 & 16 & 0.18 & 14 \\
$\mathrm{Ni}_{\mathrm{YCo}}$ & 1.75 & 1044 & 0.35 & 270 & 21 & 6 \\
$\mathrm{Y}_{2} \mathrm{Fe}_{17}$ & 1.45 & 1385 & 0.11 & 890 & 56 & 6 \\
$\mathrm{GdZn}_{\mathrm{LaCo}}$ & 0.51 & 631 & 0.15 & 660 & 15 & 6 \\
$\mathrm{YFe}_{11} \mathrm{Ti}$ & 1.24 & 930 & 0.7 & 240 & 9.4 & 6 \\
$\mathrm{YFe}_{2}$ & 1.45 & 270 & 1.9 & 26 & 3.9 & 6 \\
$\mathrm{Mn}_{5} \mathrm{Ge}$ & 1.06 & 1285 & 0.2 & 680 & 31 & this work \\
$\mathrm{MnAlGe}$ & 0.53 & 534 & 0.68 & 120 & 5.8 & "- \\
\hline & 1.00 & 553 & 0.3 & 360 & 8.2 & "- \\
\hline
\end{tabular}

As another demostration of the method, single crystals of 5 further ferromagnets of topical interest have been produced and investigated in this work. These are $\mathrm{LaCo}_{13}, \mathrm{YFe}_{11} \mathrm{Ti}, \mathrm{YFe}_{2}, \mathrm{Mn}_{5} \mathrm{Ge}_{3}$, and MnAlGe. The starting metals were all $99.99 \%$ pure, except $\mathrm{Y}$ and $\mathrm{La}$ (both $99.95 \%$ ) and $\mathrm{Ge}$ (99.999\%). For $\mathrm{YFe}_{11} \mathrm{Ti}$ the elements were mixed in the stoichiometric proportion, whereas for $\mathrm{Mn}_{5} \mathrm{Ge}_{3}$ and MnAlGe a $5 \%$ at. surplus of $\mathrm{Mn}$ was put in, to compensate for evaporation during the melting. Finally, the starting compositions for $\mathrm{LaCo}_{13}$ and $\mathrm{YFe}_{2}$ were distinctly off-stoichiometric, $\mathrm{La}_{0.189} \mathrm{Co}_{0.811}$ and $\mathrm{Y}_{0.45} \mathrm{Fe}_{0.55}$, as required for the reactive flux technique. In all cases the initial mixture of metals was melted in an induction furnace under a slight excess pressure (1.5 bars) of argon. The resulting ingots, weighing between 20 and $30 \mathrm{~g}$, were put into zirconia crucibles, these were placed into quartz tubes and sealed off under a pressure of about 0.3 bar of argon.

The quartz ampoules were subsequently placed into a resistive tube furnace for remelting. For the line compounds, $\mathrm{YFe}_{11} \mathrm{Ti}, \mathrm{Mn}_{5} \mathrm{Ge}_{3}$, and MnAlGe, the procedure consisted in heating the ingots up to slightly above the melting temperature, waiting for 5 minutes, and cooling down slowly, at a rate of $0.5^{\circ} \mathrm{C} / \mathrm{min}$, to room temperature. The La-Co ingot was heated up to $1310^{\circ} \mathrm{C}$, kept at that temperature for $5 \mathrm{~min}$, then cooled down to $1130^{\circ} \mathrm{C}$ at a rate of $5^{\circ} \mathrm{C} / \mathrm{min}$, kept at that temperature for two weeks, and finally quenched in water. The ingot for the production of $\mathrm{YFe}_{2}$ was heated up to $1200^{\circ} \mathrm{C}$, kept there for $5 \mathrm{~min}$, cooled down to $1125^{\circ} \mathrm{C}$ at a rate of $5^{\circ} \mathrm{C} / \mathrm{min}$, then more slowly (at $2^{\circ} \mathrm{C} / \mathrm{h}$ ) down to $900^{\circ} \mathrm{C}$, kept there for two weeks, then quenched in water.

The final ingots were broken up and large single- crystalline grains were extracted. The strained surface layer of the grains was etched off electrolytically in phosphoric acid. The final compositions were determined from energy-dispersive x-ray analysis and found to correspond to the desired stoichiometries. The singlecrystallinity of the grains was checked by back-scattering Laue x-ray diffraction.

The crystals of cubic symmetry $\left(\mathrm{LaCo}_{13}\right.$ and $\left.\mathrm{YFe}_{2}\right)$ were roughly spherical in shape and up to $3 \mathrm{~mm}$ in diameter; magnetic measurements were performed on unoriented, free to rotate crystals. In the case of tetragonal $\left(\mathrm{YFe}_{11} \mathrm{Ti}\right.$ and $\left.\mathrm{MnAlGe}\right)$ or hexagonal $\left(\mathrm{Mn}_{5} \mathrm{Ge}_{3}\right)$ symmetry, the crystals were needle-shaped, up to $3 \mathrm{~mm}$ long and $1 \mathrm{~mm}$ thick, the long dimension being parallel to the highsymmetry axis [001] as verified by x-ray diffraction. For magnetic measurements these crystals were glued onto sample holders so that the field would be applied along [001], which is the easy magnetization direction in all three compounds.

Magnetization curves were measured using a commercial magnetometer (Quantum Design PPMS-14) in static magnetic fields of up to $5 \mathrm{~T}$ at temperatures ranging from $5 \mathrm{~K}$ to just above the Curie points (up to $1000 \mathrm{~K}$ in the case of $\mathrm{LaCo}_{13}$ ). Extra measurements on $\mathrm{LaCo}_{13}$ were carried out between room temperature and $1273 \mathrm{~K}$ using a high-temperature magnetometer (Lake Shore VSM Model 740) in a field of up to $2 \mathrm{~T}$.

Spontaneous magnetization was determined by linear extrapolation to zero internal field, as shown with dashed lines on the upper curve in Fig. 1a. This technique works well at low temperatures but becomes ambiguous near the Curie point. Thus, the curves taken at $T=291 \mathrm{~K}$ and $295 \mathrm{~K}$ look similar in Fig. 1a. Yet, the first temper- 

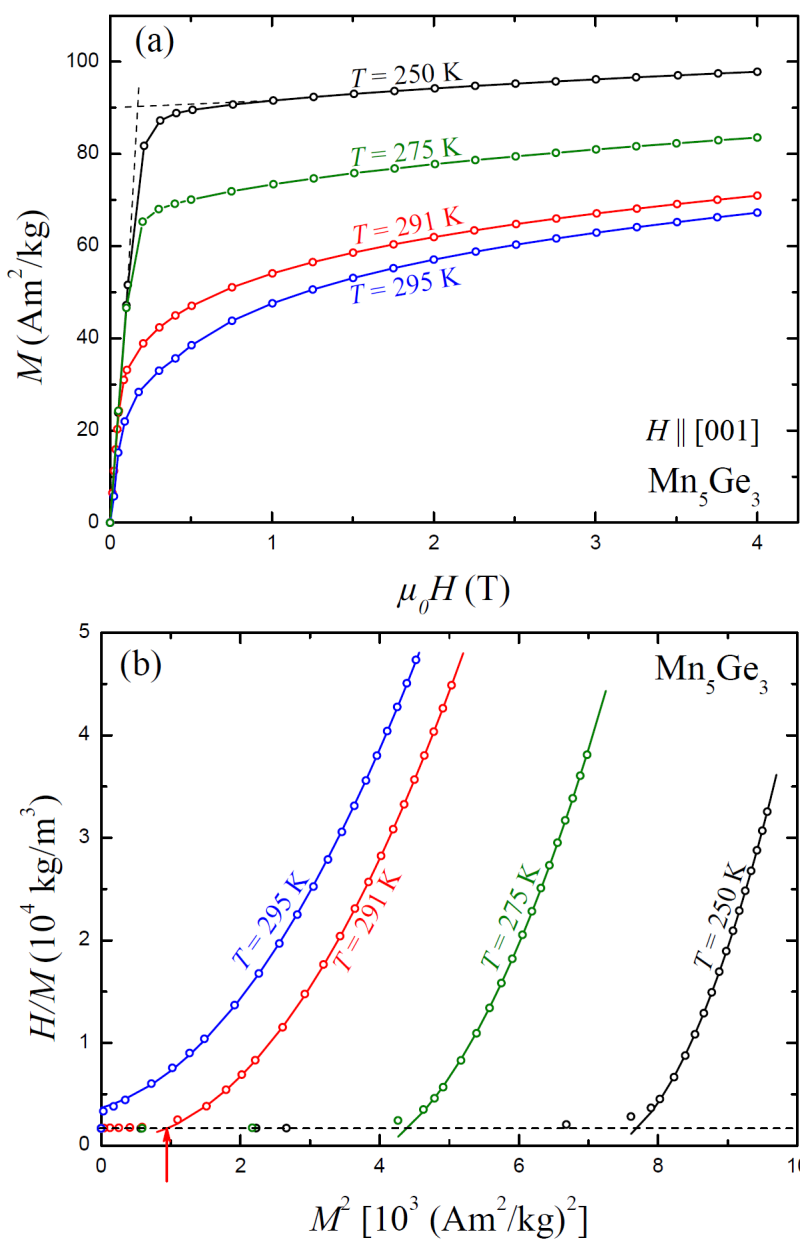

FIG. 1. Representative magnetization curves of $\mathrm{Mn}_{5} \mathrm{Ge}_{3}$ (a) and the corresponding Belov-Arrott plots (b).

ature lies below the Curie point, while the other one is above $T_{C}$. The difficulty was overcome by using near $T_{C}$ a standard procedure due to Belov \& Goryaga ${ }^{15}$ (sometimes attributed to Arrott ${ }^{16}$ ) and based on Landau's theory of second-order phase transitions, ${ }^{17}$ adapted for ferromagnets by Ginzburg. ${ }^{18}$ The technique consists in presenting the magnetization curves as $H / M$ versus $M^{2}$, as shown for $\mathrm{Mn}_{5} \mathrm{Ge}_{3}$ in Fig. 1b. The data points situated well above the (dashed) demagnetization line, $H / M \equiv N \rho \approx 2 \times 10^{3} \mathrm{~kg} / \mathrm{m}^{3}$, were fitted to quadratic polynomials and the abscissas of the crossing-points of the fits with the demagnetization line were interpreted as values of $M_{s}^{2}$. This is indicated with an arrow for the curve at $T=291 \mathrm{~K}$. The $295 \mathrm{~K}$ curve is clearly distinct; here $M_{s}^{2}<0$, so $M_{s}$ is not a real quantity. The BelovArrott plots were used to determine $M_{s}$ at $T>260 \mathrm{~K}$ for $\mathrm{Mn}_{5} \mathrm{Ge}_{3}, T>450 \mathrm{~K}$ for MnAlGe, $T>480 \mathrm{~K}$ for $\mathrm{YFe}_{11} \mathrm{Ti}, T>500 \mathrm{~K}$ for $\mathrm{YFe}_{2}$, and $T>1220 \mathrm{~K}$ for $\mathrm{LaCo}_{13}$. The results are shown as open circles in Fig. 2.

The data points were fitted to Eq. (4) with $p=5 / 2$ and $\beta=1 / 3$; the fits are shown in Fig. 2 as continuous lines. The best-fit values of the parameters $M_{0}$,

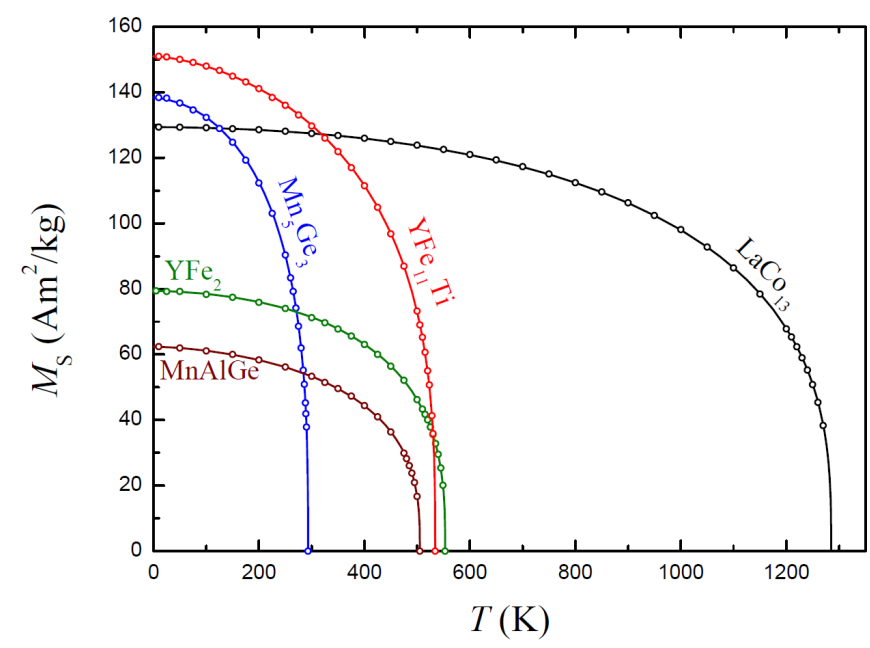

FIG. 2. Temperature dependence of spontaneous magnetization. Open circles indicate values deduced from experiment and continuous lines are fits to Eq. (4).

$T_{C}$, and $s$ are collected in the lower part of Table I; xray densities were used to convert $M_{0}$ into amperes per meter. The obtained $T_{C}$ values are in reasonable agreement with the literature: $T_{C}=1290 \mathrm{~K}$ for $\mathrm{LaCo}_{13},{ }^{19}$ $296 \mathrm{~K}$ for $\mathrm{Mn}_{5} \mathrm{Ge}_{3},{ }^{20} 503 \mathrm{~K}$ for $\mathrm{MnAlGe},{ }^{21} 538 \mathrm{~K}$ for $\mathrm{YFe}_{11} \mathrm{Ti}^{22}$ and $560 \mathrm{~K}$ for $\mathrm{YFe}_{2} \cdot{ }^{23}$ (We find that the latter value should be reduced to $556 \mathrm{~K}$, as follows from a careful analysis of the data presented in Fig. 3 of Ref. 23; this agrees yet better with the result of this work for $\mathrm{YFe}_{2}, T_{C}=553 \mathrm{~K}$.) We do not aim at giving here an exhaustive survey of published $T_{C}$ data. Just for $\mathrm{LaCo}_{13}$ we would like to point out that the only other reported value, $T_{C}=1318 \mathrm{~K},{ }^{24}$ is definitely too high.

Finally, the values of $D$ and $A$ found from Eqs. (3) and (5) are presented in the $5^{\text {th }}$ and $6^{\text {th }}$ columns of Table I. A few remarks are due at this point.

(i) Both $D$ and $A$ are referred to $T=0$; they are not to be compared with room-temperature data. However, as ground-state properties, they should be amenable to DFT calculations.

(ii) For a non-cubic ferromagnet the value of $A$ (or $D$ ) given in Table I should be understood as the geometric mean of the principal values of the corresponding tensor, $A=\left(A_{1} A_{2} A_{3}\right)^{1 / 3}$. For a domain wall of particular orientation one of the tensor components is relevant, not the geometric mean.

(iii) Values of $A$ deduced from the properties of domain walls (such as thickness or energy density) are affected by the poor knowledge of anisotropy constants, all of them usually neglected but the leading one. Such data cannot be regarded as accurate, even if they were obtained from low-temperature measurements.

We are therefore left with two main sources of information: inelastic neutron scattering and density functional calculations. The comparison (see Table II) is essentially limited to iron and nickel, for which low-temperature neutron scattering data are available. 
TABLE II. Spin-wave stiffness of iron and nickel, $D\left(m e V \AA^{2}\right)$, obtained by various techniques.

\begin{tabular}{lll}
\hline source & $\mathrm{Fe}$ & $\mathrm{Ni}$ \\
\hline neutron scattering & $307^{a}$ & $400^{b}, 593^{c}$ \\
DFT calculation & $220^{d}, 247^{e}, 250^{f}$ & $700^{d}, 739^{e}, 756^{f}$ \\
this work & 270 & 660 \\
\hline
\end{tabular}

${ }^{a}$ Ref. 25.

${ }^{b}$ Ref. 26.

${ }^{c}$ Ref. 27.

${ }^{d}$ Ref. 28.

${ }^{e}$ Ref. 29.

${ }^{f}$ Ref. 30 .

The situation for iron appears benign, but one learns little from the comparison. Perhaps, that the first calculated value, ${ }^{28} \mathrm{D}=220 \mathrm{meV \AA} \AA^{2}$, is somewhat too low.

The numbers for nickel are more telling. So one learns to mistrust the values of $D$ deduced from neutron spectra. The reason is that $D$, defined as the curvature of the dispersion curve at $q=0$, is not directly accessible to neutron spectroscopy. Instead, data are collected at nonzero $q$ and fitted to an expression with two or three adjustable parameters, one of them being $D$. The procedure is apparently numerically unstable, as disparate values of $D$ were obtained in Refs. 27 and 26. (The situation is worse for the factor of $q^{4}$ - neither its sign nor order of magnitude could be found out.) It is not inconceivable that the observed part of the spectrum could be also reproduced by using the spin-wave stiffness obtained in this work, $D=660 \mathrm{meV}^{2}$, and a suitably chosen coefficient of the quartic term. It is not clear at this point if our result should be regarded as an underor an over-estimation.

For cobalt, there are no low-temperature neutron data; the often-cited value, ${ }^{31} D=510 \mathrm{meV}^{2}$, was deduced from a spectrum taken at room temperature. It is instructive, though, to compare our result for Co with the one obtained by Pathenet, ${ }^{32} D=580 \mathrm{meV}^{2}$, by fitting his own $M_{s}(T)$ data to Bloch's law. Our value is significantly higher, $D=890 \mathrm{meV}^{2}$, but the corresponding $s$ was obtained in Ref. 6 by fitting to Eq. (4) a combined data set, whose low-temperature part $(T<300$ $\mathrm{K}$ ) had been taken from Ref. 32. It is rather peculiar that two so different $D$ 's should be deduced from practically the same experimental data. (All the more so as the values obtained for iron are very close: our 274 meV $\AA^{2}$, rounded off to $270 \mathrm{meVA}^{2}$ in Table I, versus Pauthenet's $276 \mathrm{meV}^{2}$, rounded off to $280 \mathrm{meV} \AA^{2}$ in Ref. 32.) It should be noted, though, that the data set used in Ref. 6 was an extended one; it covered the entire interval from $T=4.2 \mathrm{~K}$ to the Curie point; all those data were fitted successfully to Eq. (4) with the standard exponents, $p=5 / 2$ and $\beta=1 / 3$. On the contrary, in Ref. 32 the fitting interval was narrowed down to $100 \mathrm{~K}<T<287 \mathrm{~K}$, excluding deliberately the data collected between $4.2 \mathrm{~K}$ and $100 \mathrm{~K}$. The exclusion of the low-temperature data points is in contradiction with the asymptotic, low-temperature character of the Bloch law and it may have compromised the result. In any case, Pauthenet himself regarded the obtained factor of $T^{3 / 2}$ for Co as rather approximate; so one reads in Ref. 32: $a_{3 / 2} \sim 1.5 \times 10^{-6}\left(\mathrm{~K}^{-3 / 2}\right)$. The thence derived stiffness value, $D=580 \mathrm{meV}^{2}$, should be therefore viewed as a less accurate one.

To conclude, we propose a new way of deducing the spin-wave stiffness $D$ and the exchange stiffness $A$ of a ferromagnet from its spontaneous magnetization. The main advantage of the new technique is that the entire $M_{s}(T)$ dependence is used (with $0<T<T_{C}$ ) rather than just its low-temperature part.
1 S.S.P. Parkin, M. Hayashi, and L. Thomas, Science 320, 190 (2008).

2 E. Lifshitz, J. Phys. USSR 8, 337 (1944).

${ }^{3}$ F. Keffer, Spin Waves. In: Handbuch der Physik, edited by S. Flügge (Springer, Berlin, 1966), Vol. 18/2, p. 1.

4 C. Herring and C. Kittel, Phys. Rev. 81, 869 (1951).

${ }^{5}$ W. Döring, Mikromagnetismus. In: Handbuch der Physik, edited by S. Flügge (Springer, Berlin, 1966), Vol. 18/2, p. 341.

6 M.D. Kuz'min, Phys. Rev. Lett. 94, 107204 (2005).

7 M. Fries, Z. Gercsi, S. Ener, K.P. Skokov, and O. Gutfleisch, Acta Mater. 113, 213 (2016).

8 A. Edström, M. Werwiński, D. Iuşan, J. Rusz, O. Eriksson, K.P. Skokov, I.A. Radulov, S. Ener, M.D. Kuz'min, J. Hong, M. Fries, D.Yu. Karpenkov, and O. Gutfleisch, Phys. Rev. B 92, 174413 (2015).

9 M.D. Kuz'min, K.P. Skokov, I. Radulov, C.A. Schwöbel, S. Foro, W. Donner, M. Werwiński, J. Rusz, E. DelczegCzirjak, and O. Gutfleisch, J. Appl. Phys. 118, 053905 (2015).
10 M.D. Kuz'min, D. Givord, and V. Skumryev, J. Appl. Phys. 107, 113924 (2010).

11 A.V. Andreev, S. Yoshii, M.D. Kuz'min, F.R. de Boer, K. Kindo, and M. Hagiwara, J. Phys.: Condens. Matter 21, 146005 (2009).

12 E.A. Tereshina and A.V. Andreev, Intermetallics 18, 641 (2010).

13 M.D. Kuz'min, A.S. Chernyshov, V.K. Pecharsky, K.A. Gschneidner, Jr., and A.M. Tishin, Phys. Rev. B 73, 132403 (2006).

14 M.D. Kuz'min and A.M. Tishin, Phys. Lett. A 341, 240 (2005).

15 K.P. Belov and A.N. Goryaga, Fiz. Met. Metalloved. 2, 3 (1956).

16 A. Arrott, Phys. Rev. 108, 1394 (1957).

17 L.D. Landau, Phys. Z. Sowjetunion 11, 26 (1937).

18 V.L. Ginzburg, Zh. Eksp. Teor. Fiz. 17, 833 (1947).

19 W.A.J.J. Velge and K.H.J. Buschow, J. Appl. Phys. 39, 1717 (1968).

${ }^{20}$ G. Kappel, G. Fischer, and A. Jaéglé, Phys. Stat. Sol. (a) 
34, 691 (1976).

21 T. Kamimura, H. Ido, and K. Shirakawa, J. Appl. Phys. $\mathbf{5 7}, 3255$ (1985).

22 S.A. Nikitin, I.S. Tereshina, V.N. Verbetskii, and A.A. Salamova, Phys. Solid State 40, 258 (1998).

${ }^{23}$ V. Paul-Boncour, M. Escorne, A. Mauger, M. Latroche, and A. Percheron-Guegan, J. Appl. Phys. 79, 4253 (1996).

${ }^{24}$ H. Ido, J.C. Sohn, F. Pourarian, S.F. Cheng, and W.E. Wallace, J. Appl. Phys. 67, 4978 (1990).

${ }^{25}$ C.-K. Loong, J.M. Carpenter, J.W. Lynn, R.A. Robinson, and H.A. Mook, J. Appl. Phys. 55, 1895 (1984).

26 P.W. Mitchell and D.McK. Paul, Phys. Rev. B 32, 3272
(1985)

27 J.W. Lynn and H.A. Mook, Phys. Rev. B 23, 198 (1981).

28 M.D. Kuz'min, M. Richter, and A.N. Yaresko, Phys. Rev. B 73, 100401 (2006).

29 N.M. Rosengaard and B. Johansson, Phys. Rev. B 55, 14975 (1997)

30 M. Pajda, J. Kudrnovský, I. Turek, V. Drchal, and P. Bruno, Phys. Rev. B 64, 174402 (2001).

31 G. Shirane, V.J. Minkiewicz, and R. Nathans, J. Appl. Phys. 39, 383 (1968).

32 R. Pauthenet, J. Appl. Phys. 53, 8187 (1982). 